\title{
Simulation of Discharge Characteristics of Ferroelectric Ceramics Transducer Based on Simulink
}

\author{
Gu Lin , Zhang He \\ ZNDY of Ministerial Key Laboratory \\ Nanjing University of science \& technology
}

\author{
Zhang Xiaojing \\ Nanjing University of science \& technology
}

\begin{abstract}
To analyze the depolarization and discharge effect of ferroelectric ceramics, the physical model of ferroelectric ceramics depolarized by the vertical shock wave is established. Equivalent circuit of the depolarization is built. The mechanical structure of the transducer is designed. The shock wave initiated by the explosive-driven flyer is numerically calculated. The discharge characteristics of the ferroelectric ceramic transducer are simulated. Simulation in MATLAB demonstrates that the power level of the ferroelectric ceramic transducer could reach up to megawatt. In the case of shortcircuit load, current waveform is approximately square wave. Meanwhile, small inductive and resistive composite load resents the damping oscillation waveform.
\end{abstract}

Keywords-Ferroelectric ceramics, Transducer, Discharge characteristics; Simulation

\section{INTRODUCTION}

Ferroelectric ceramics depolarize and release electric charge by the shock wave, which can be used as explosiveto-electrical power[1]. The ferroelectric ceramic transducer can discharge high power pulse on the load. As the portable high-power pulsed power of high energy density, small size, low cost and strong independence, the transducer has broad application in the fields of industry and national defense[2].

In this paper, the mechanical structure of the transducer in which shock wave is loaded by explosive-driven flyer is designed. The discharge characteristics of ferroelectric ceramics transducer are simulated by SIMULINK module of the software MATLAB.

\section{PHYSICAL MODEL}

PZT95/5 $\left(\mathrm{PbZr}_{0.95} \mathrm{Ti}_{0.05} \mathrm{O}_{3}\right)$ is a typical kind of ferroelectric ceramics. Its solid solution component is neighbouring between the boundaries of ferroelectric phase and the anti-ferroelectric phase in the phase diagram. As the transition of ferroelectric phase and anti-ferroelectric phase caused by the pressure or the electric field, they are also named phase-transition ferroelectric ceramics. PZT95/5 is converted to the metastable ferroelectric phase in the same polarization orientation by the applied electric field. Under the pressure, metastable ferroelectric phase will return to the stable anti-ferroelectric phase, and release stored charge during the process of depolarization. As the result of these discharge characteristics, PZT95/5 can be used in high power ferroelectric ceramics transducer.

The vertical shock wave load model of ferroelectric ceramics means that the direction of the shock wave is perpendicular to the polarization of ferroelectric ceramics.
Yuanji He and Jinmei Du have studied the discharge characteristics of ferroelectric ceramics with short-circuit load $[3,4]$. The electrical parameter of the load in this research is inductive and resistive.

Figure. 1 (a) is the schematic diagram of Ferroelectric transducer. Figure. 1 (b) is the equivalent circuit. Ferroelectric ceramics transducer is equivalent to a constant current source with fixed value capacitor in parallel. During the process of shock wave propagation, capacitance and conductance will be fluctuated because of the change of wave-interface area.

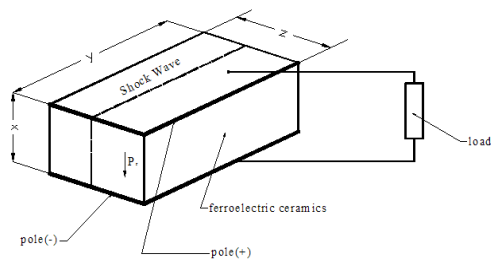

(a)

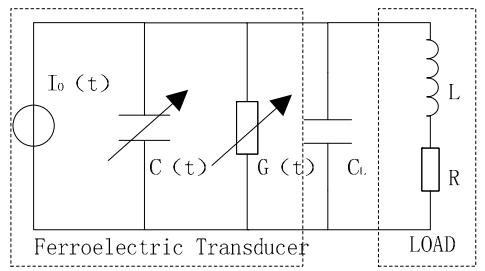

(b)

Figure 1. Schematic diagram and equivalent circuit of ferroelectric transducer

In the circuit model, ferroelectric ceramics are equivalent to the time-varying current source $I(t)$ 、 $C(t)$ and $G(t)$ in parallel. Considering the medium relaxation effect, the function of $I(t)$ is derived as the following formula, in which $t_{s c}$ is the relaxation time.

$$
I_{0}(t)= \begin{cases}P_{r} A\left[1-\exp \left(-t / t_{s c}\right)\right] / t_{0} & t \leq t_{0} \\ P_{r} A \exp \left(-\left(t-t_{0}\right) / t_{s c}\right)\left[1-\exp \left(-t / t_{s c}\right)\right] / t_{0} & t>t_{0}\end{cases}
$$

In the function above, $A=y z$. The calculations of $C(t)$ and $G(t)$ are detailed in the reference [4,5].

\section{DESIGN OF FERROELECTRIC TRANSDUCER}

\section{A. Preparation of ferroelectric ceramics}

The fabrication process of PZT95/5 is shown in Figure. 2. Pure metal oxides are used as raw materials. All the necessary raw materials are weighed at the stoichiometric ratio, additional $3 \%$ of $\mathrm{P}_{\mathrm{b}} \mathrm{O}$ raw material is added . Afrer 24 hours' ball-milling, it is dryed and burned at $700-800^{\circ} \mathrm{C}$ for 
the secondary ball-milling. Then, 5 percents of PVA solution is added. After this, it is molded under the pressure more than $140 \mathrm{MPa}$, complete plastic-removal at $600^{\circ} \mathrm{C}$. At last, PZT95/5 is sintered at $1250-1300^{\circ} \mathrm{C}$.

\section{B. Mechanical design of ferroelectric transducer}

Ferroelectric transducer includes the detonator, detonator support, body shell, shock flyer (aluminum), PZT module. Ferroelectric ceramics (PZT ceramic) are placed in the PZT holder which is filled by epoxy as the buffering and electrical insulating material. After the detonator sets off explosives in the high explosive chamber, the resulting explosive shock wave drives the flyer to impact ferroelectric ceramics, makes it depolarized and release a lot of electric charge. It can adjust the flyer speed by regulating the volume of charge chamber, explosive amount and the flyer's flying distance of the transducer, fulfilling the loading parameter control.

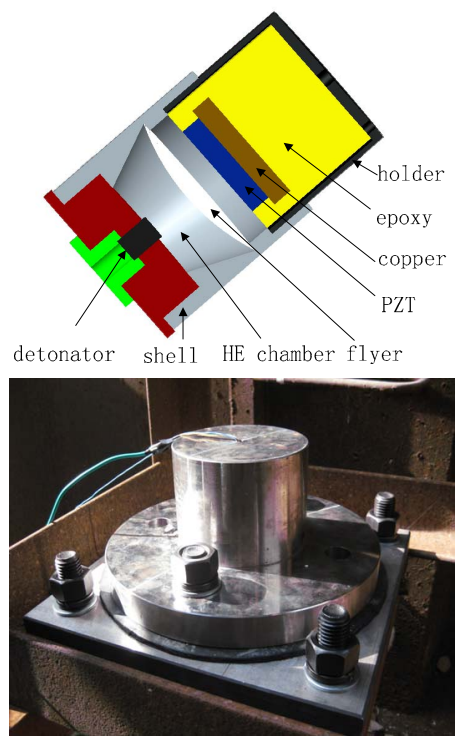

Figure 3. Mechanical structure of the transducer and outside view

As the speed of the flyer increases, the impact force at the shock wave front increases. Meanwhile, the initial shock wave velocity in the ferroelectric ceramics increases. It is calculated in reference [6] that charge length of $4 \mathrm{~mm}$ can produce impact pressure more than $10 \mathrm{GPa}$, and initial shock wave velocity of $4744 \mathrm{~m} / \mathrm{s}$ in the ferroelectric ceramics. The initial pressure of the phase transition is about $0.5 \mathrm{GPa}$, the complete phase transition pressure is between $1 \mathrm{GPa}$ to $3 \mathrm{GPa}$ Therefore, the shock wave loaded by flyer impact not only can fully meet the load conditions of phase transition of ferroelectric ceramics, but also reduce the volume of the transducer. So the design requirement of miniaturization could be achieved.

\section{Discharge CHARACTERISTIC Simulation}

The equivalent circuit of the ferroelectric ceramics depolarization discharge was simulated by SIMULINK module of software MATLAB, and the discharge characteristic of the ferroelectric ceramics explosive power transducer was studied. Compared to the numerical simulation, the simulation model based on SIMULINK is more visuable. It is not necessary to write a large number of the programs or the formula functions. It avoids the complexity and versatility of the numerical simulation.

Because of the function $i=C(d u / d t)$, nonlinear capacitance component can be expressed by a controlled current source. The current source is controlled by the voltage across it, build nonlinear capacitance mode (Figure.4), The module includes a controlled current source, voltage meter, differential, product, the prepared S function of time-varying capacitor and some other modules.

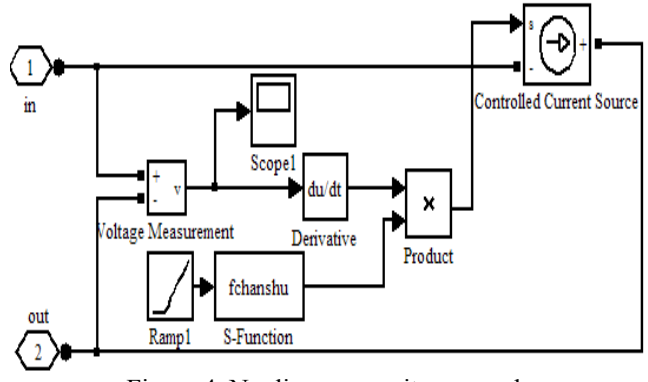

Figure 4. Nonlinear capacitance mode

According to $R(t)=1 / G(t)$, the simulation established the resistance model to displace the conductivity model. Nonlinear resistance component can be expressed by a controlled voltage source. The voltage source is controlled by the current.The silulation had built nonlinear resistance mode (Figure.5). The $\mathrm{S}$ module in the figure is $\mathrm{S}$ function of $R(t)$. On this basis, it establishes the equivalent circuit simulation mode (Figure.6) of ferroelectric ceramics explosive power transducer. In the mode, "sI", "sC", and "sG" are respectively ferroelectric ceramics time-varying current sources, capacitance, and conductance of the $\mathrm{S}$ function.

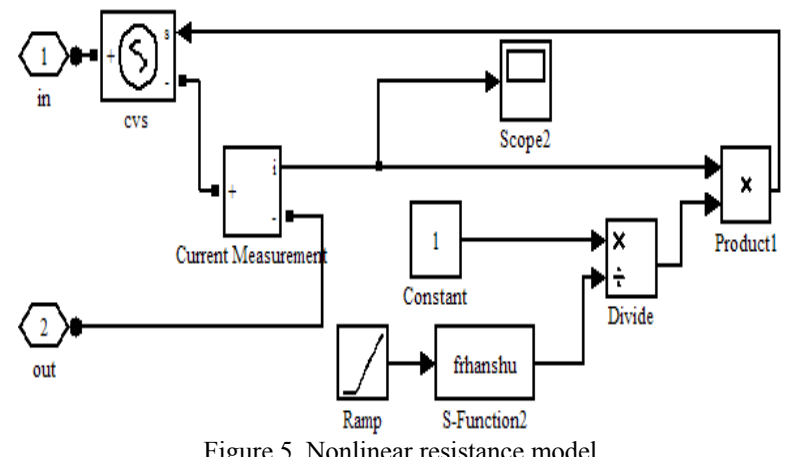

The ferroelectric ceramic's properties, size parameters and load parameters are shown in Table. 1.

Small load electrical parameters ( $R=1200 m \Omega$, $L=0.3 \mu \mathrm{H}$ ) and short-circuit load simulation current waveform under two different load are shown in Figure. 7. Figure. 8 is the simulation waveforms of the transducer with matching capacitor $\left(C_{L}\right.$, value is $\left.40 n F\right)$. 


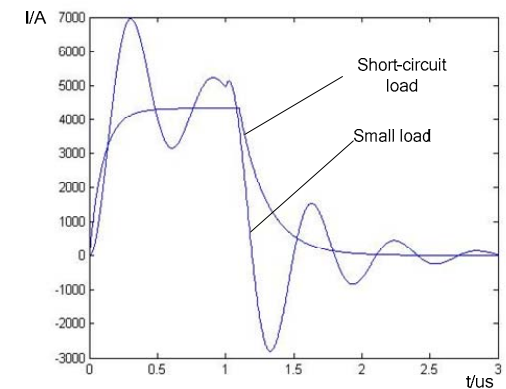

Figure 7. Simulation waveform on different load

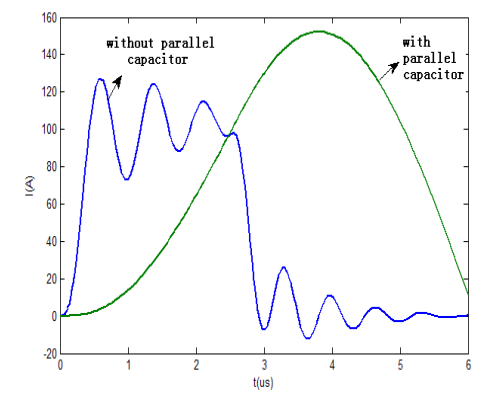

Figure 8. Simulation waveform with parallel capacitor

Simulation results show that ferroelectrics electrical explosion transducer discharge current waveform is approximately a square wave under the short-circuit load, damping oscillation waveform appears under small perceptual and small resistive complex load. In ferroelectric depolarization discharge process the voltage can reach several $\mathrm{kV}$, therefore ferroelectric electrical explosive transducer's power level can achieve megawatt.

\section{SUMMARIES}

A new design of compacting pulsed high-power resourse is provided. Shock wave loading mechanical devices of ferroelectric electrical explosion transducer is designed. Its working principle and equivalent circuit are introduced. According to circuit models, the discharge characteristics under short-circuit load and small load are simulated. The power of transducer can achieve megawattclass. With short-circuit load current waveform is approximately a square wave, meanwhile damping oscillation waveform would appear with small perceptual and small resistive complex load.

\section{REFERENCES}

[1] Lysne P C, Percival C M. Electric energy generation by shock compression of ferroelectric ceramics: Normal-mode response of PZT95/5[J]. Apply Phys, 1975, 46(4):1519-1525

[2] HE Yuan-ji, ZHANG Ya-zhou, LI Chuan-lu.High Power Pulse Supplier of PZT95/5 Ferroelectric Ceramics for Charging of Nanofarad Capacitors[J].HIGH VOLTAGE ENGINEERING, 2004,30 (4) : 34-35

[3] Mock W, Jr, Holf W H. Pulse Charging of Nanofarad Capacitors from the Shock Depoling of PZT 56/44 and PZT95/ 5 Ferroelectric Ceramic[J]. J Appl Phys, 1978, 49:5846

[4] HE Yuan-ji, ZHANG Ya-zhou, LI Chuan-lu,WANG Hong-gang. The Numerical Simulation of Electric Response ofPZT95/5 Ferroelectric Ceramics Subjected to Shock Loading[J].CHINESE JOURNAL OF HIGH PRESSURE PHYSICS.2000, 14(3): 189-194

[5] DU Jin-mei, Zhang Yi. Large Current out-put of PZT 95/5 Ferroelectric Ceramics under Shock Loading[J]. ACTA PHYSICA SINICA, 2006, 55(5):2584-2588

[6] ZHANG Shou-zhong. Explosion and Impact Dynamics[M].Beijing: Weapon Industry Press, 1993.4
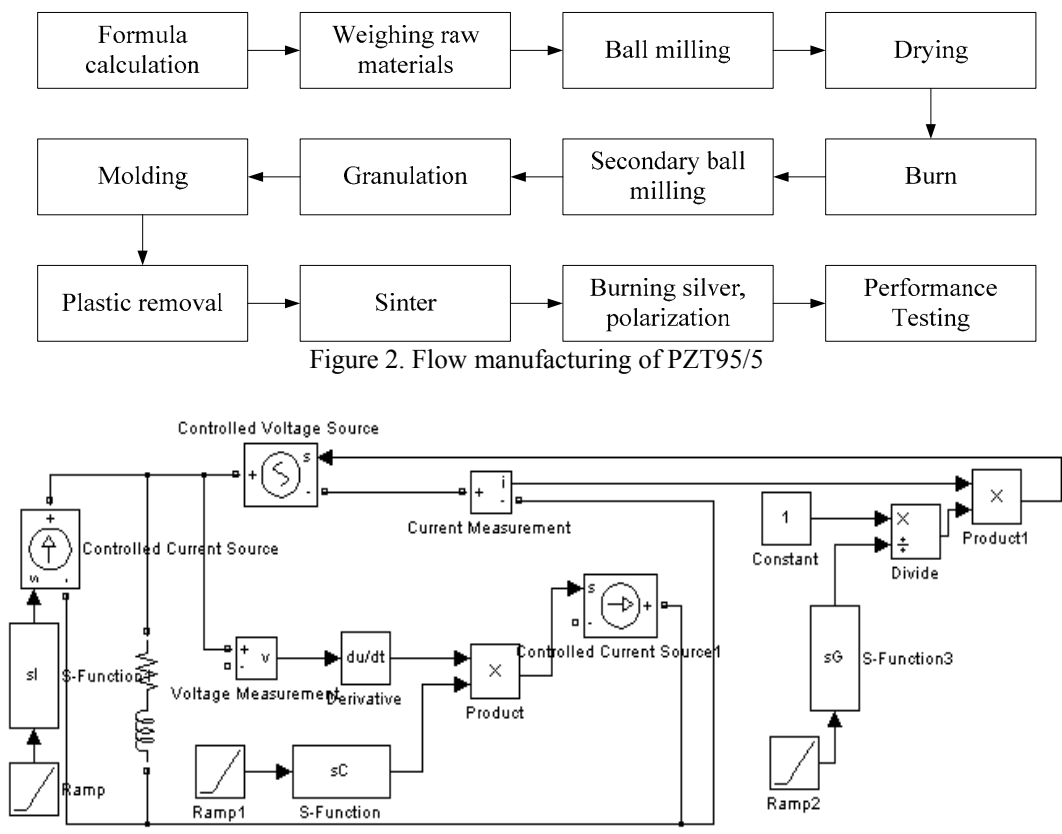

Figure 6. Equivalent circuit simulation mode 
TABle I. PARAMETERS OF THE SIMULATION MODEL

\begin{tabular}{cccc}
\hline Parameter & Value & Parameter & Value \\
\hline$P_{r}\left(u C / \mathrm{cm}^{2}\right)$ & $33 \sim 35$ & $x(\mathrm{~mm})$ & 10 \\
$h_{1}(F / m)$ & $1.6 \times 10^{-8}$ & $y(\mathrm{~mm})$ & 35 \\
$h_{2}(F / m)$ & $0.9 \times 10^{-8}$ & $z(\mathrm{~mm})$ & 4 \\
$g_{1}(1 / \Omega \cdot m)$ & $2.5 \times 10^{-6}$ & $R(\mathrm{~m} \Omega)$ & $0 / 1200$ \\
$g_{2}(1 / \Omega \cdot m)$ & $2.5 \times 10^{-3}$ & $L(\mu H)$ & $0 / 0.3$ \\
$t_{s c}(u s)$ & 0.3 & $n$ & 100 \\
\hline
\end{tabular}

